\title{
Certeza vocacional y factores de carrera en estudiantes de dos universidades del noroeste de México
}

\section{Vocational Certainty and Career Factors in Students of two Northwestern Universities of Mexico}

Daniel González Lomelí

Laura Fernanda Barrera Hernández

María de los Ángeles Maytorena Noriega

María de los Ángeles Fuentes Vega

Universidad de Sonora

\section{Resumen}

La elección de carrera es un proceso de toma de decisiones fundamental para el desarrollo futuro de los adolescentes, inmersos en un mundo de continua transformación y donde el desarrollo de habilidades de formación continua está presente. Los objetivos de este estudio fueron explorar un modelo que explique la Certeza Vocacional (CV) a partir de Factores de Carrera (FC), cognoscitivos y emocionales, en estudiantes de dos instituciones del noroeste de México, además de comparar los perfiles de CV y FC de ambas muestras por variables sociodemográficas (como género, tipo de opción de la carrera, condición laboral, haber realizado cursos o materias de orientación vocacional durante el bachillerato, universidad y carrera en la que estudia). Con base en el modelamiento por ecuaciones estructurales y la participación de 248 estudiantes de nivel superior, se encontró que los factores de carrera, necesidad de información sobre la carrera y ansiedad ante la elección de carrera, explican 55\% de la variabilidad de la certeza vocacional. Se encontraron diferencias en los perfiles vocacionales entre los estudiantes de ambas universidades. Estos datos contribuyen a la teoría en relación con la importancia de contar con medidas pertinentes para la toma decisión de carrera.

Palabras clave: certeza vocacional, educación superior, factores de carrera, modelamiento estructural, noroeste de México.

Nota del autor

Daniel González Lomelí, Universidad de Sonora. Retorno Rancho Bonito, \#65, Rancho Bonito Residencial, C. P. 83174, Hermosillo, Sonora, México. Tel.: 6622 2592205. Correo electrónico: daniel. lomeli@unison.mx. Laura Fernanda Barrera Hernández, Universidad de Sonora.

Esta investigación fue financiada por el Consejo de Ciencia y Tecnología de México a través de la convocatoria de Ciencia Básica 2011/167235.

María de los Ángeles Maytorena Noriega, Instituto Tecnológico de Sonora.

María de los Ángeles Fuentes Vega, Instituto Tecnológico de Sonora. 


\begin{abstract}
Choosing a career is a fundamental decision-making process with implications for the future development of adolescents. Adolescents are immersed in a continually transforming world where skill development is continuous. The main objective of this study was to explore a causal model of vocational certainty $(\mathrm{CV})$ predicted by cognitive and emotional career factors (FC) in students from two different institutions in northwestern Mexico. Further, CV and FC profiles of each sample were compared. Sociodemographic variables (such as gender, type of career option, employment status, having completed courses or subjects of vocational guidance during high school, university and career in which he studies) were also included in the model. Based on structural equation modeling ( $n=248$ higher education students), results showed that career factors were informed by career information and anxiety. In turn, career choice explained $55 \%$ of vocational certainty variability. Additionally, differences were found between the vocational profiles in students from the two different universities. These results contribute evidence highlighting the importance of measuring relevant variables in the study of career decision making.
\end{abstract}

Keywords: vocational certainty, higher education, career factors, structural modeling, northwest Mexico.

A principios de este siglo, el panorama mundial se ha caracterizado por un cambio social más rápido e intenso, con efectos sobre la familia, la economía, el mundo del trabajo, la política, la cultura y, en general, la existencia cotidiana de las personas en cualquier esfera o contexto social (Fernández, 2003). Tal panorama apunta hacia un tipo de cultura profesional que implica interés y capacidad de análisis de los problemas y sucesos más importantes y significativos de la sociedad en que vivimos.

En un estudio con bachilleres se encontraron diferencias significativas en el estado de la decisión vocacional, tanto por edad como por sexo, así como en la preferencia por las carreras (Canto, 2000). Las mujeres prefirieron carreras relacionadas con Sociales y Biológicas, mientras que los hombres prefirieron carreras relacionadas con Biológicas y Matemáticas.
El autor concluyó que, aparentemente, los estudiantes se deciden por una carrera, aunque no están completamente seguros de lo adecuado de su decisión.

En nuestro medio, esta elección se realiza durante el nivel bachillerato, sin embargo, también sería el caso de los estudiantes universitarios que sienten que no corresponden sus estudios con sus habilidades, con sus intereses o con sus expectativas laborales $y$ prefieren desertar (Fuentes, 2010). La deserción escolar es un fenómeno que se presenta con mayor intensidad en los primeros semestres de la licenciatura, como lo han identificado Velarde, González y Camarena (2013). En este sentido, León (2016) realizó un estudio acerca de la deserción en estudiantes universitarios del sur de Sonora, encontró que $31 \%$ de los estudiantes de nuevo ingreso en 2014 había desertado para 
el segundo año de universidad; 18\% desertó en el primero año y $13 \%$ en el segundo.

En México existe un índice considerable de abandono en los estudios superiores (INEGI, 2012), las cifras de deserción escolar en hombres y mujeres oscilan entre $16.5 \%$ y $12.4 \%$, respectivamente. Tenemos que sólo se gradúa $25 \%$ de quienes asisten a este nivel, ya que de 36 millones de jóvenes que conforman este país (31.2\% de la población total), $18.75 \%$ son estudiantes de educación superior.

Fuentes-Vega (2016) verificó que 227 egresados de un plantel del subsistema de bachilleres solicitaron su ingreso a la Universidad de Sonora y construyó su perfil de ingreso: encontró que la población de mujeres representó $58.1 \%$, en calificación de la preparatoria $51.1 \%$ de los estudiantes obtuvo un promedio menor a 85 (las mujeres destacan sobre los hombres), en la puntuación obtenida en el examen de ingreso a la universidad EXHCOBA (el máximo a alcanzar es de 190 puntos), se observó que 50.7\% obtuvo una puntuación menor a 133 puntos (aquí sobresalen los hombres). En cuanto al tipo de estudiante, se reportó que $84.6 \%$ son regulares; referente al estatus, $89.9 \%$ se reporta activo, $4.8 \%$, inactivo y $5.3 \%$, suspendido. Tanto la puntuación en el EXHCOBA (.201) como el promedio de preparatoria (.456) presentan una correlación positiva y significativa, con el promedio del primer semestre; el promedio de preparatoria presenta también una correlación positiva y significativa (.350) con la variable de tipo de estudiante.
Hou, Wu y Lui (2013) señalan que la presión severa del empleo provoca que un creciente número de estudiantes encuentren dificultades en la toma de decisiones de carrera. En relación con lo anterior, Gati y Levin (2014) refieren que la indecisión de la carrera puede ser la razón principal por la que la gente asiste con orientadores vocacionales o consejeros de carrera, asimismo, derivado de los esfuerzos por identificar e investigar las causas de las dificultades de toma de decisión de la carrera, se han desarrollado instrumentos de medida de la indecisión de carrera, de los cuales se recomienda obtener resultados que permitan promover la eficacia y la eficiencia de la orientación profesional y facilitar la toma de decisión de la carrera de los clientes.

En 1990, Chartrand, Robbins, Morrill y Boggs, bajo la premisa de que tanto la información que se obtiene sobre la carrera, como componentes personal-emocionales interactúan, bien para facilitar o bien para inhibir los procesos de toma de decisiones de carrera, diseñaron y probaron un modelo que incluye dimensiones de factores de información con los componentes: necesidad de información de carrera y necesidad de autoconocimiento, además factores emocionales-personales que incluyen autoestima, ansiedad en la elección de carrera e indecisión generalizada. Los cinco componentes fueron puestos a prueba vía autoreporte a partir de análisis factorial confirmatorio. Se definen a continuación: 
Autoestima es la evaluación característica que hace una persona de sí misma. Juega un papel central en la formación del autoconcepto vocacional. Ansiedad en la elección de carrera fue definida como el nivel de ansiedad reportado en el proceso de toma de decisión vocacional. Este factor se ha identificado como un antecedente de la indecisión vocacional. Indecisión generalizada se definió como la falta de habilidad de las personas para tomar decisiones cuando las condiciones están presentes. La habilidad para tomar decisiones juega un papel importante en el proceso de toma de decisión de carrera.

El cuarto factor, Necesidad de información de carrera, se refiere a la percepción de una persona sobre la necesidad de obtener información y experiencia real acerca de diferentes ocupaciones antes de realizar un compromiso vocacional. Es identificada como una de las mejores categorías diagnósticas en problemas de toma de decisión de carrera. Mientras que el factor Necesidad de autoconocimiento se define como la necesidad de cada persona de autodefinirse y descubrirse y es de las más importantes en diversos modelos de elección de carrera; una persona con esta característica denota una identidad confusa con una pobre claridad de sus cualidades personales, sus capacidades e intereses.

Después de los análisis confirmatorios correspondientes, el modelo de Chartrand y colaboradores (1990), antes descrito, se conformó por cuatro factores; el factor autoestima es el que no se constituyó. Así el instrumento diseñado por los autores, el Inventario de Factores de Carrera, permite discriminar a los individuos que reportan diferentes niveles de indecisión de carrera. El modelo resultante posee validez convergente y los índices de consistencia interna son superiores a .73 .

En México Aguilar, Pacheco, Andrade, Vargas, Gutiérrez y colaboradores (1992), basándose en el modelo de Chartrand y compañeros (1990), ampliaron el Inventario de Factores de Carrera (la versión de cuatro factores), incluyendo dos factores: la escala de autoeficacia definida como la apreciación del individuo sobre sus propias capacidades e intereses relacionados con determinada carrera y la escala de indecisión vocacional, que los autores desarrollaron como una medida de contraste. El Inventario Ampliado de Factores de Carrera (IAFC) cuenta con dos versiones, una para estudiantes de bachillerato y otra para estudiantes de licenciatura.

Posteriormente, González y Maytorena (2005), con base en la propuesta de Aguilar y compañeros (1992), encontraron en una muestra de 229 estudiantes universitarios de Psicología y Químico-biólogo, que los factores de carrera: autoconocimiento, información sobre la carrera, autoeficacia, ansiedad en la elección de carrera e indecisión generalizada, afectan de manera positiva y significativa (.57) a la seguridad vocacional (entendida como la seguridad que el estudiante tiene en la carrera que ha elegido) y el esfuerzo académico, sólo que en menor grado (.29). Y en 2007, los mismos autores, con una muestra de 296 estudiantes de bachillerato y 176 
de licenciatura, observaron que la variable que predice en mayor medida la certeza vocacional es autoconocimiento del estudiante, en el caso de los de bachillerato, y en los de licenciatura fue el factor información sobre la carrera (González, \& Maytorena, 2007).

Mientras que Velarde y González (2013), parten del objetivo de relacionar factores personales (factores de carrera) y sociales con la certeza vocacional en estudiantes de universidades públicas y privadas a partir del modelamiento estructural, para posteriormente contrastar sus hallazgos con los contenidos de la materia de Orientación Educativa (OV) durante el bachillerato. Con una muestra de 392 estudiantes, encontraron que las variables latentes factores sociales y necesidades de carrera explican $23 \%$ de la varianza de la certeza vocacional. Y que los contenidos programáticos de las asignaturas de OV favorecen sólo parte de las necesidades cognoscitivas (necesidad de información sobre la carrera y necesidad de autoeficacia) del estudiantado y dejan de lado los factores emocionales (ansiedad ante la elección de carrera e indecisión generalizada) y los factores sociales.

Recientemente, Fuentes-Vega (2016) empleó el Inventario Ampliado de Factores de Carrera en su versión para bachillerato con estudiantes de último semestre, los análisis de regresión realizados arrojaron que los factores de carrera predictores de la certeza vocacional son necesidad de información, necesidad de autoeficacia, indecisión generalizada y ansiedad en la elec- ción de carrera $\left(\mathrm{R}^{2}=.36\right)$, en relación con otros factores que intervienen en la toma de decisión para elección de carrera, como lo es la familia, por ejemplo, se identificó que los indicadores que predicen la certeza vocacional $\left(\mathrm{R}^{2}=.59\right)$ fueron la interferencia de los padres, variable definida como la influencia de los padres sobre la toma de decisión de los hijos, y la falta de compromiso de los padres, atribuida a la falta de interés real o baja importancia atribuida a la elección de carrera.

Con relación a los factores de carrera que integran el modelo de Aguilar y colaboradores (1992), donde la autoeficacia se vislumbra como uno de los factores importantes en el proceso de elección, se reportan en los siguientes párrafos investigaciones sobre la autoeficacia en la elección de carrera.

Por ejemplo, en el estudio de Burns, Jasinski, Dunn y Fletcher (2013), los resultados indicaron que las evaluaciones de los servicios de apoyo académico se relacionaron positivamente con los niveles de autoeficacia en la toma de decisiones profesionales. Isik (2012) examinó la relación entre la autoeficacia en la decisión de carrera, la ansiedad rasgo y afecto positivo y negativo en 249 estudiantes de primer año que se inscribieron en los cursos de preparación de inglés en una universidad de Turquía, sus edades variaron entre 17 y 23 años. Al finalizar el análisis de datos, se tienen como hallazgos principales que las correlaciones entre la autoeficacia, decisión de carrera y ansiedad rasgo y afecto positivo $y$ negativo fueron estadísticamente significativas. 
En específico, la autoeficacia en la decisión de carrera estuvo inversamente relacionada con la ansiedad rasgo, con un efecto moderado; positivamente relacionada con el afecto positivo, con un tamaño del efecto moderado e inversamente relacionado con el afecto negativo. Los resultados del análisis de regresión múltiple indicaron que la ansiedad rasgo, el afecto positivo y negativo, afectan en conjunto $21 \%$ de la varianza en la autoeficacia de toma de decisiones de carrera. Sin embargo, la ansiedad rasgo y el afecto positivo fueron los únicos predictores significativos.

Además, Kim e investigadores (2013) realizaron un estudio en Corea del Sur, acerca de la influencia de los padres en la indecisión de carrera de 302 estudiantes, los resultados del estudio muestran que hay un efecto del conflicto de independencia de la madre en la indecisión; es decir, altos niveles de conflicto de independencia de la madre indican bajos niveles de indecisión y de indecisión de carrera. La relación entre indecisión general e indecisión de carrera también fue significativa. Otros hallazgos fueron que la indecisión general es mediadora entre independencia conflictiva de la madre e indecisión de carrera; y que el efecto de la independencia conflictiva de la madre en la indecisión de carrera sería más grande que el efecto del apego hacia la madre en la indecisión de carrera.

En el estudio de Hou y compañeros (2013), los resultados mostraron que tanto la calidez emocional de los padres y la conciencia, correlaciona negativa y significativamente con las dificultades en la toma de decisiones de carrera y la relación entre calidez emocional de los padres y conciencia, fue positiva y significativa. En el modelo de mediación, observaron que la calidez emocional de los padres tenía un efecto indirecto sobre las dificultades en la toma de decisiones de carrera de los estudiantes universitarios a través de la mediación del estado de conciencia (conscientiousness). En el modelo integrado, este efecto indirecto fue moderado por un ambiente intelectual y cultural orientado a la familia. Los resultados mostraron que el efecto negativo de calidez emocional de los padres en dificultades en la toma de decisiones de carrera fue significativo, al igual que el efecto mediador de calidez emocional de los padres en la conciencia, y conciencia en las dificultades en la toma de decisiones de carrera. Después de añadir el mediador, el efecto directo de calidez emocional de los padres sobre las dificultades en la toma de decisiones de carrera se redujo.

Hsieh y Huang (2014) en Taiwán analizaron las relaciones existentes entre el nivel socioeconómico de la familia, la personalidad proactiva y la autoeficacia en la toma de decisión de carrera. Participaron 336 personas de 21 años de edad en promedio. Los resultados confirman que el nivel socioeconómico de la familia está positiva y significativamente asociado con la autoeficacia en la toma de decisiones de carrera; los estudiantes que reportan niveles socioeconómicos más altos reportan también mayor confianza en su habilidad para finalizar satisfactoriamente tareas de toma de decisiones de carrera. Los resultados además confirman que 
existe una relación positiva y significativa entre la personalidad proactiva y la autoeficacia en la toma de decisiones de carrera.

Ahora bien, existen otros factores que están relacionados con la toma de decisión vocacional, factores que pueden ser sociales, medioambientales o de género, por ejemplo, son factores que pueden fungir en un momento dado como predictores de decisiones vocacionales. En torno a esto, algunos investigadores han buscado la relación de dichos factores con la certeza vocacional o con la toma de decisión vocacional, a saber, la investigación realizada por Bulgare1li, Rivera y Fallas (2016), en la cual buscaban analizar el proceso vocacional de los estudiantes en condición de logro y rezago académico en estudiantes de bachillerato en Química de la universidad de Costa Rica, partiendo del enfoque evolutivo de Super. Fue un estudio de caso colectivo, en el cual participaron cuatro estudiantes, dos de cada condición académica; utilizaron tres instrumentos para la recogida de datos, tales como entrevista, sesiones a profundidad y observación, el análisis se hizo mediante el contenido categorial temático. Los autores concluyeron con su investigación que en el proceso de la toma de decisión vocacional de los cuatro casos estudiados presentaban diferencias entre ambas condiciones académicas, en donde la condición de logro estaba más inclinada a tener un mejor rendimiento vocacional, pero no era un predictor de éste. En la condición de rezago observaron que se presentaba mayor dificultad en las diversas etapas vocacionales. Los autores concluyeron que es importante estar atentos del apoyo académico, vocacional, personal y social de los jóvenes que se encuentren en cualquiera de las dos condiciones académicas analizadas, además de considerar sus diferencias y similitudes en torno al proceso vocacional.

De igual manera, están también aquellas investigaciones en las cuales se considera relevante estudiar la participación de las mujeres en el ámbito de la educación superior, tal es el caso de la investigación realizada por De Garay y Del Valle (2012), quienes señalan que la inclusión de una perspectiva de género en la educación superior es un factor que no puede ni debe postergarse, es importante que se trabaje en el tema de la inclusión de género desde la toma de decisión vocacional, ya que es común observar que en las áreas de ciencias exactas e ingenierías la participación de la mujer representa un mínimo porcentaje, dando a entender que dichas áreas son campos de estudio masculinizados; asimismo, todo esto se relaciona con la participación de las mujeres en puestos directivos, una vez que ingresan al ámbito laboral.

Por su parte, Macías (2016) ha identificado discrepancias en la participación de la matrícula universitaria entre hombres y mujeres, tanto en los ámbitos nacional como internacional, para lo cual plantea tres retos a resolver por las instituciones de educación superior y por las instancias de gobierno: a) las diferencias de participación por niveles, b) las ausencias por campo de estudios y c) la vinculación al mercado laboral. 
Los objetivos de este estudio fueron explorar un modelo que explique la $\mathrm{CV}$ a partir de $\mathrm{FC}$ cognoscitivos y emocionales, en estudiantes de dos instituciones del noroeste de México, además de comparar los perfiles de $\mathrm{CV}$ y FC con base en variables sociodemográficas (como género, tipo de opción de la carrera, condición laboral, haber realizados cursos o materias de orientación vocacional durante el bachillerato y carrera que estudia) de ambas muestras. Para lo cual se puso a prueba un modelo teórico $\mathrm{y}$, a través del modelamiento por ecuaciones estructurales (MEE), se estimó el poder explicativo de factores psicológicos que son considerados por la literatura nacional e internacional, pertinentes en la comprensión de la certeza vocacional. Lo anterior con el fin de buscar generalizar los resultados obtenidos con muestras de estudiantes de escuelas públicas y privadas (Mariñez, \& González, 2013), de bachilleres y de licenciatura (González, \& Maytorena, 2007) y de bachilleres (FuentesVega, 2016) de la ciudad de Hermosillo, Sonora, y generar información orientada a facilitar la optimización los recursos destinados a la atención del estudiantado, en busca de aportar al desempeño óptimo en su formación profesional.

Se hipotetiza que la certeza vocacional recibe efectos directos y negativos, tanto de los factores de carrera cognoscitivos (necesidad de autoconocimiento, necesidad de autoeficacia y necesidad de información de carrera), como de los factores de carrera emocionales (ansiedad en la elección de carrera e indecisión generalizada).

\section{Método}

\section{Participantes}

La muestra disposicional estuvo conformada por 248 estudiantes de nivel superior, 150 de una universidad pública de la ciudad de Hermosillo, Sonora, de las licenciaturas en Psicología $(n=75)$ y Negocios y Comercio Internacional $(n=75)$, y 98 estudiantes de una universidad pública de Cd. Obregón, Sonora, de las licenciaturas en Psicología $(n=62)$ y Administración $(n=36)$. Del total de la muestra, $67.1 \%$ son mujeres, $95.9 \%$ estaban solteros, su media de edad fue de 19.9 años $(D E=1.98)$ y su promedio de calificaciones en bachillerato fue de $86.85(D E=6.59) ; 68.8 \%$ reportó haber cursado una materia de orientación vocacional en el bachillerato y sólo $14.6 \%$ indicó haber llevado un curso de orientación vocacional. Los estudiantes que trabajan (26.9\%) indican hacerlo $6.8 \mathrm{~h}$ a la semana $(D E=13)$ en promedio. El $83 \%$ de los estudiantes cursa su primera opción de licenciatura y las tres actividades extraclase que más realizan fueron actividades de estudio (25.8\%), deporte (18.1\%) e idiomas (9.3); sobresale que $38.7 \%$ de los estudiantes no realiza ninguna actividad además de asistir a clases. Los estudiantes provienen de diferentes subsistemas de bachillerato: CBTIS (14.5\%), COBACH (35.9\%), CECYTES (13.3\%), escuelas particulares $(22.2 \%)$ y otras $(14.1 \%)$.

\section{Instrumentos}

Inventario Ampliado de Factores de Carrera (IAFC) de Aguilar (Aguilar, Peña, Pacheco, \& 
De la Paz, 1993), el cual fue validado a través de un análisis factorial confirmatorio por MEE con una muestra de estudiantes universitarios (González, \& Maytorena, 2005) y está constituido por seis escalas: a) tres escalas de información (cognoscitivas) de tipo Likert con seis opciones de respuesta desde 1 (totalmente en desacuerdo) a 6 (totalmente de acuerdo): necesidad de autoconocimiento, necesidad de información sobre la carrera y necesidad de autoeficacia; b) por dos escalas emocionales, de tipo diferencial semántico, con seis anclajes: indecisión generalizada y ansiedad en la elección de carrera y; c) por una escala tipo Likert para medir certeza vocacional (la seguridad que el estudiante tiene en la carrera que ha elegido) con seis opciones de respuesta desde 1 (totalmente en desacuerdo) a 6 (totalmente de acuerdo).

Ejemplo de reactivos:

Reactivo de la dimensión de necesidad de autoconocimiento (cinco reactivos).

1) Para que pueda decidir si sigo o no estudiando la carrera que actualmente estudio, todavía necesito contestar la siguiente pregunta: ¿Cuáles son mis valores personales?

Reactivo de la dimensión de necesidad de información sobre la carrera (cinco reactivos).

2) Para que pueda decidir si sigo o no estudiando la carrera que actualmente estudio, todavía necesito informarme sobre los planes de estudio de varias carreras en diferentes universidades y los requisitos de ingreso.
Reactivo de la dimensión de necesidad de autoeficacia (cuatro reactivos).

3) No creo tener la capacidad necesaria para seguir con la carrera que estoy estudiando.

Reactivo de la dimensión de certeza vocacional (seis reactivos).

4) Tengo dificultades para decidir si sigo o no estudiando la carrera que actualmente estudio.

Reactivo de la dimensión ansiedad en la elección de carrera (tres reactivos).

5) Cuando pienso en si sigo o no estudiando la carrera que actualmente estudio me siento:

a) Tenso No tenso

Reactivo de la dimensión indecisión generalizada (siete reactivos).

6) En general tomar decisiones me resulta:

a) Confuso No confuso

\section{Procedimiento}

El inventario ampliado de factores de carrera se aplicó en los horarios y salones de clase de ambas instituciones de educación superior, con la participación voluntaria e informada de los estudiantes, previa firma de la carta de consentimiento informado y la autorización de los coordinadores de cada licenciatura.

\section{Análisis de datos}

Con los datos obtenidos de la aplicación del IAFC en ambas instituciones, se realizaron análisis de frecuencias para las variables descriptivas sociodemográficas de género, estado civil, trabajo y actividades extraclase; y se obtuvieron promedios estadísticos, con sus respectivas desviaciones estándar para variables como edad y promedio de bachillerato. Se realizaron análisis 
de varianza para probar diferencias entre ambas muestras; también, análisis de correlación entre las seis escalas y análisis de regresión para probar un modelo de $\mathrm{CV}$ y los factores de carrera como predictores. Finalmente, se realizó un análisis factorial confirmatorio por Modelamiento de Ecuaciones Estructurales (MEE) y se obtuvieron los indicadores de bondad de ajuste (Bentler, 2006).

\section{Resultados}

En la tabla 1 se presentan los estadísticos descriptivos de las escalas, así como los resultados del análisis de consistencia interna realizado a las escalas que conforman el IAFC después del MEE, en ella se observa que las escalas poseen valores $\alpha$ de .69 a .85, por lo cual se asume que todas las escalas son confiables.

Tabla 1.

Media y consistencia interna de las escalas del IAFC y de certeza vocacional después del MME

\begin{tabular}{|c|c|c|c|c|c|c|}
\hline Escalas/Reactivos & $N$ & Min. & Max. & $M$ & $D E$ & $\alpha^{*}$ \\
\hline Autoconocimiento & 245 & 1 & 6 & 3.13 & & .82 \\
\hline Decidir valores personales & & 1 & 6 & 3.11 & 1.75 & \\
\hline Decidir quién soy & & 1 & 6 & 2.71 & 1.67 & \\
\hline Decidir clase de persona & & 1 & 6 & 3.33 & 1.68 & \\
\hline Decidir cosas importantes para mí & & 1 & 6 & 3.39 & 1.69 & \\
\hline Autoeficacia & 248 & 1 & 6 & 2.67 & & .69 \\
\hline No capacidad de seguir carrera & & 1 & 6 & 1.61 & 1.10 & \\
\hline Dificultan materias & & 1 & 6 & 3.11 & 1.27 & \\
\hline Dificultan aspectos de la profesión & & 1 & 6 & 2.70 & 1.37 & \\
\hline Faltan habilidades & & 1 & 6 & 3.29 & 1.37 & \\
\hline Información sobre la carrera & 245 & 1 & 6 & 2.70 & & .81 \\
\hline Información sobre planes & & 1 & 6 & 2.31 & 1.50 & \\
\hline Necesidad platicar con personas & & 1 & 6 & 2.83 & 1.54 & \\
\hline Necesidad pedir consejos & & 1 & 6 & 2.04 & 1.30 & \\
\hline Necesidad conocer oportunidad de trabajo & & 1 & 6 & 3.58 & 1.68 & \\
\hline Necesidad de orientación sobre la carrera & & 1 & 6 & 2.78 & 1.69 & \\
\hline Ansiedad en la elección & 245 & 1 & 6 & 2.55 & & .85 \\
\hline Tenso al pensar si sigo o no carrera & & 1 & 6 & 2.32 & 1.42 & \\
\hline Preocupado al pensar si sigo o no & & 1 & 6 & 2.62 & 1.58 & \\
\hline Ansioso al pensar si sigo o no carrera & & 1 & 6 & 2.72 & 1.55 & \\
\hline Indecisión generalizada & 246 & 1 & 6 & 2.96 & & .76 \\
\hline Tomar decisiones es confuso & & 1 & 6 & 2.72 & 1.33 & \\
\hline Tomar decisiones es difícil & & 1 & 6 & 3.05 & 1.41 & \\
\hline $\mathrm{Al}$ tomar decisiones soy seguro & & 1 & 6 & 2.54 & 1.23 & \\
\hline Al tomar decisiones soy preocupado & & 1 & 6 & 3.53 & 1.40 & \\
\hline Certeza vocacional & 245 & 1 & 6 & 5.05 & & .73 \\
\hline Dificultad decidir si sigo estudiando & & 1 & 6 & 4.80 & 1.52 & \\
\hline Continuaré hasta terminar la carrera & & 1 & 6 & 5.51 & 1.08 & \\
\hline Carrera actual es la que quiero estudiar & & 1 & 6 & 5.10 & 1.36 & \\
\hline Actualmente hago lo que quería hacer & & 1 & 6 & 4.83 & 1.52 & \\
\hline
\end{tabular}

*Alfa de Cronbach fue utilizada como indicador de confiabilidad 
Se efectuaron comparaciones por género de las variables medidas en el Inventario de Factores de Carrera; con relación a variables generales como el promedio de bachillerato, donde las estudiantes autoreportan un promedio de bachillerato $(M=87.81)$ significativamente mayor $\left(t_{239}=-3.28, p<.001\right)$ que los estudiantes hombres $(M=84.90)$. Mientras que las estudiantes mujeres presentan una mayor indecisión generalizada $(M=2.93)$ en comparación con los hombres $\left(M=2.69 ; t_{244}=\right.$ $-2.11, p<.03)$.

En cuanto a la certeza vocacional, los estudiantes que cursan la licenciatura que consideraron su primera opción reportan una mayor certeza vocacional $(M=5.26)$ que los estudiantes reubicados $(M=4.85)$ que cursan una segunda opción; esta diferencia es significativa $\left(t_{245}=-2.92, p<.004\right)$. En cuanto a los factores de carrera, se encontró que los estudiantes reubicados muestran una mayor necesidad de autoconocimiento $(M=3.91)$ que los estudiantes no reubicados $\left(M=3.47 ; t_{245}=\right.$ $2.12, p<.03)$.
En cuanto a los factores emocionales, los estudiantes de la muestra que no trabajan presentan mayor indecisión generalizada ( $M$ $=2.93)$ en comparación con los estudiantes que trabajan $(M=2.66)\left(t_{240}=-2.21, p<.02\right)$. Los estudiantes reubicados muestran mayor ansiedad ante la elección de carrera $(M=3.08)$ que los estudiantes que cursan su primera opción de licenciatura $\left(M=2.46 ; t_{244}=2.74, p<.007\right)$.

Al comparar a los estudiantes que cursaron una asignatura de orientación vocacional, con los que no cursaron dicha asignatura en el bachillerato, no se encontraron diferencias significativas en certeza vocacional, ni en ninguno de los factores de carrera, sean cognoscitivos o emocionales. Resultados similares se observaron al comparar a los estudiantes que llevaron un curso de orientación fuera de la escuela, con los que no lo llevaron.

Con relación a la institución educativa en la que estudian, se encontró una diferencia significativa en el factor necesidad de autoeficacia $\left(t_{246}=2.70, p<.007\right)$, mayor en los estudiantes de la universidad del centro del estado $(M=2.80)$ que los estudiantes de la universidad al sur del estado $(M=2.48)$.

Tabla 2.

Diferencias entre los estudiantes de las dos universidades

\begin{tabular}{|c|c|c|c|c|c|c|c|c|}
\hline & \multicolumn{2}{|c|}{ UNISON } & \multicolumn{2}{|c|}{ ITSON } & \multirow[b]{2}{*}{$g l$} & \multirow[b]{2}{*}{$t$} & \multirow[b]{2}{*}{$p$} & \multirow[b]{2}{*}{$d$ Cohen } \\
\hline & $M$ & $D E$ & $M$ & $D E$ & & & & \\
\hline Necesidad de autoconocimiento & 3.55 & 1.124 & 3.56 & 1.36 & 246 & -.05 & .959 & 0.00 \\
\hline Necesidad de autoeficacia & 2.80 & .873 & 2.48 & .983 & 246 & 2.70 & .007 & 0.34 \\
\hline Necesidad de información de la carrera & 2.77 & 1.071 & 2.59 & 1.314 & 246 & 1.18 & .239 & 0.15 \\
\hline Ansiedad en la elección & 2.64 & 1.308 & 2.44 & 1.423 & 245 & 1.09 & .274 & 0.14 \\
\hline Indecisión generalizada & 2.92 & .785 & 2.76 & .950 & 246 & 1.50 & .133 & 0.18 \\
\hline Certeza vocacional & 5.12 & .838 & 5.30 & .833 & 246 & 1.66 & .098 & 0.21 \\
\hline
\end{tabular}


Se efectúo una comparación entre los estudiantes de Psicología de ambas instituciones educativas (tabla 3) y se encontraron diferencias significativas respecto a necesidad de autoeficacia, necesidad de información de la carrera y ansiedad en la elección; los estudiantes de la universidad del centro del estado presentaron medias más elevadas, es decir, mayor necesidad de autoeficacia y de información de la carrera, además mayor ansiedad ante la elección de carrera. También se observó diferencia respecto a certeza vocacional, en la cual los estudiantes de la universidad del sur de Sonora reportaron mayor seguridad vocacional.

\section{Tabla 3.}

Diferencias entre los estudiantes de Psicología

\begin{tabular}{lcccccccccc}
\hline & \multicolumn{1}{c}{ UNISON } & & \multicolumn{2}{c}{ ITSON } & & & & \\
\cline { 2 - 3 } & $M$ & $D E$ & & $M$ & $D E$ & $g l$ & $t$ & $p$ & $d$ Cohen \\
\hline Necesidad de autoconocimiento & 3.66 & 1.12 & & 3.38 & 1.36 & 118.28 & 1.31 & .190 & 0.22 \\
Necesidad de autoeficacia & 2.87 & .89 & & 2.35 & .92 & 135 & 3.37 & .001 & 0.57 \\
Necesidad de información de la carrera & 2.71 & 1.09 & & 2.28 & 1.24 & 135 & 2.16 & .033 & 0.36 \\
Ansiedad en la elección & 2.88 & 1.37 & & 2.19 & 1.38 & 135 & 2.90 & .004 & 0.50 \\
Indecisión generalizada & 2.94 & .77 & & 2.75 & 1.01 & 135 & 1.25 & .213 & 0.21 \\
Certeza vocacional & 5.10 & .87 & & 5.42 & .81 & 135 & -2.24 & .026 & 0.38 \\
\hline
\end{tabular}

Respecto a la comparación entre los de negocios, no se observaron diferencias estudiantes de Administración y los estudiantes significativas (tabla 4).

Tabla 4.

Diferencias entre los estudiantes de Administración y negocios

\begin{tabular}{|c|c|c|c|c|c|c|c|c|}
\hline & \multicolumn{2}{|c|}{ UNISON } & \multicolumn{2}{|c|}{ ITSON } & \multirow[b]{2}{*}{$g l$} & \multirow[b]{2}{*}{$t$} & \multirow[b]{2}{*}{$p$} & \multirow[b]{2}{*}{$d$ Cohen } \\
\hline & $M$ & $D E$ & $M$ & $D E$ & & & & \\
\hline Necesidad de autoconocimiento & 3.43 & 1.11 & 3.86 & 1.33 & 109 & -1.79 & .076 & 0.35 \\
\hline Necesidad de autoeficacia & 2.73 & .85 & 2.70 & 1.05 & 109 & .134 & .894 & 0.03 \\
\hline Necesidad de información de la carrera & 2.83 & 1.05 & 3.13 & 1.27 & 109 & -1.28 & .201 & 0.25 \\
\hline Ansiedad en la elección & 2.39 & 1.19 & 2.87 & 1.40 & 108 & -1.90 & .060 & 0.36 \\
\hline Indecisión generalizada & 2.91 & .80 & 2.77 & .83 & 109 & .82 & .412 & 0.17 \\
\hline Certeza vocacional & 5.14 & .80 & 5.09 & .82 & 109 & .34 & .733 & 0.06 \\
\hline
\end{tabular}

Finalmente, se efectúo una comparación de medias entre los estudiantes de Psicología y de Administración y negocios, en la cual se presentó una diferencia significativa respecto a necesidad de información en la carrera; los estudiantes de Administración y negocios reportaron mayor necesidad de información de la carrera, que los estudiantes de Psicología. 
Tabla 5.

Diferencias entre los estudiantes de Psicología y negocios-Administración

\begin{tabular}{lcccccccc}
\hline & \multicolumn{9}{c}{ Psicología } & \multicolumn{3}{c}{ Negocios } & & & & \\
\cline { 2 - 8 } & $M$ & $D E$ & $M$ & $D E$ & $g l$ & $t$ & $p$ & $d$ Cohen \\
\hline Necesidad de autoconocimiento & 3.53 & 1.24 & 3.57 & 1.20 & 246 & -.25 & .797 & .03 \\
Necesidad de autoeficacia & 2.63 & .94 & 2.72 & .91 & 246 & -.72 & .467 & .09 \\
Necesidad de información de la carrera & 2.52 & 1.17 & 2.93 & 1.13 & 246 & -2.77 & .006 & .35 \\
Ansiedad en la elección & 2.57 & 1.41 & 2.55 & 1.27 & 245 & .144 & .885 & .01 \\
Indecisión generalizada & 2.85 & .89 & 2.87 & .81 & 246 & -.139 & .890 & .02 \\
Certeza vocacional & 5.14 & .80 & 5.09 & .82 & 246 & 1.09 & .273 & .06 \\
\hline
\end{tabular}

A partir de dicho análisis y a juzgar por las puntuaciones medias reportadas por la muestra, es posible determinar que los estudiantes de Administración del ITSON, se perciben con menor certeza en la elección de carrera, con mayor necesidad de autoconocimiento, mayor necesidad de información sobre la carrera y mayor ansiedad ante la elección de carrera; y los estudiantes de Psicología de la misma insti- tución, son en promedio, quienes se manifiestan más seguros vocacionalmente, dado que reportan puntuaciones mayores que los estudiantes de la universidad en el factor certeza vocacional y más bajas en los factores de carrera, tales como necesidad de autoconocimiento, necesidad de autoeficacia, necesidad de información sobre la carrera, ansiedad ante la elección de carrera e indecisión generalizada (figura 1).

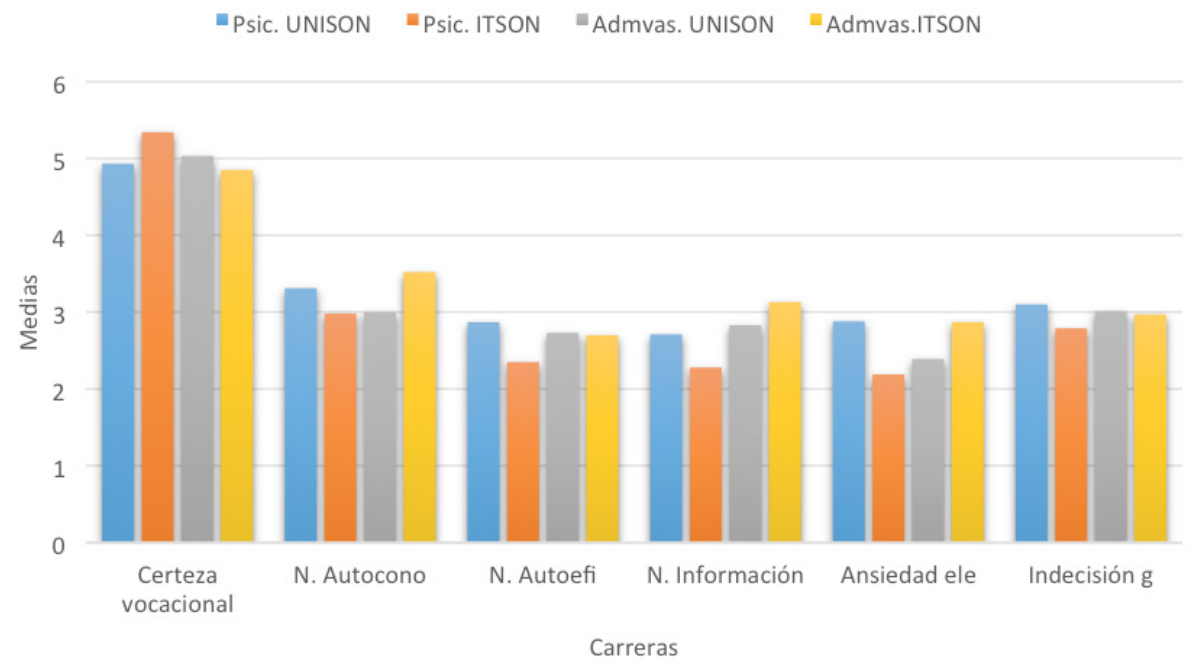

Figura 1. Comparación de medias entre certeza vocacional y los factores de carrera por licenciatura 
El resultado del análisis de correlación entre la certeza vocacional y los factores de carrera del IAFC se muestra en la tabla 6. Todas las variables se correlacionan negativamente con la certeza vocacional, como teóricamente se esperaba.
Se destacan las mayores correlaciones con la certeza vocacional y ansiedad en elección de carrera (-.50) y necesidad de información sobre la carrera (-.40), lo cual indica una valoración consistente de la relación entre estos factores.

Tabla 6.

Coeficientes de correlación entre las escalas del IAFC

\begin{tabular}{llllll}
\hline & $\begin{array}{l}\text { Necesidad de Necesidad de } \\
\text { autoeficacia }\end{array}$ & $\begin{array}{l}\text { Ansiedad } \\
\text { información } \\
\text { ante elección }\end{array}$ & $\begin{array}{l}\text { Indecisión } \\
\text { generalizada }\end{array}$ & $\begin{array}{l}\text { Certeza } \\
\text { vocacional }\end{array}$ \\
\hline $\begin{array}{l}\text { Necesidad de } \\
\text { autoconocimiento }\end{array}$ & $.19^{* *}$ & $.59^{* *}$ & $.30^{* *}$ & $.14^{*}$ & $-.22^{* *}$ \\
$\begin{array}{l}\text { Necesidad de } \\
\text { autoeficacia }\end{array}$ & --- & $.29^{* *}$ & $.32^{* *}$ & $.24^{* *}$ & $-.32^{* *}$ \\
$\begin{array}{l}\text { Necesidad de } \\
\text { información sobre }\end{array}$ & & & & & \\
$\begin{array}{l}\text { la carrera } \\
\text { Ansiedad ante la } \\
\text { elección de carrera }\end{array}$ & -- & $.40^{* *}$ & $.23^{* *}$ & $-.40^{* *}$ \\
$\begin{array}{l}\text { Indecisión } \\
\text { generalizada }\end{array}$ & & --- & $.26^{* *}$ & $-.50^{* *}$ \\
\hline
\end{tabular}

${ }^{\star} p<.05,{ }^{* *} p<.01$

Un análisis de regresión múltiple de las puntuaciones en certeza vocacional como variable dependiente es predicho por las variables ansiedad en la elección de carrera, necesidad de información sobre la carrera y necesidad de autoeficacia, con una $R$ cuadrada ajustada de .313 , lo que significa que $31 \%$ de la varianza de certeza vocacional es explicada por las variables cuyos coeficientes de regresión fueron significativos (tabla 7).

Tabla 7 .

Coeficientes estandarizados de regresión múltiple para certeza vocacional

\begin{tabular}{lcrc}
\hline \multicolumn{1}{c}{ Variables } & \multicolumn{1}{c}{ Beta } & \multicolumn{1}{c}{$t$} & \multicolumn{1}{c}{$p$} \\
\hline Necesidad de autoconocimiento & .075 & 1.129 & .260 \\
Necesidad de autoeficacia & -.153 & -2.649 & .009 \\
Necesidad de información sobre la carrera & -.261 & -3.713 & .000 \\
Ansiedad ante la elección de carrera & -.375 & -6.197 & .000 \\
Indecisión generalizada & .018 & .319 & .750 \\
\hline
\end{tabular}




\section{Modelo estructural}

Se elaboró y probó un modelo estructural por MEE para explicar la influencia que recibe la certeza vocacional de las variables que miden factores de carrera (figura 2). La certeza vocacional recibe un efecto directo y negativo de la ansiedad en la elección de carrera y de la necesidad de información sobre la carrera. La necesidad de autoconocimiento y la necesidad de autoeficacia tienen un efecto indirecto sobre la certeza vocacional a través de la necesidad de información sobre la carrera. Asimismo, se observa un efecto indirecto de la indecisión generalizada sobre la certeza vocacional a través de la ansiedad ante la elección de carrera.

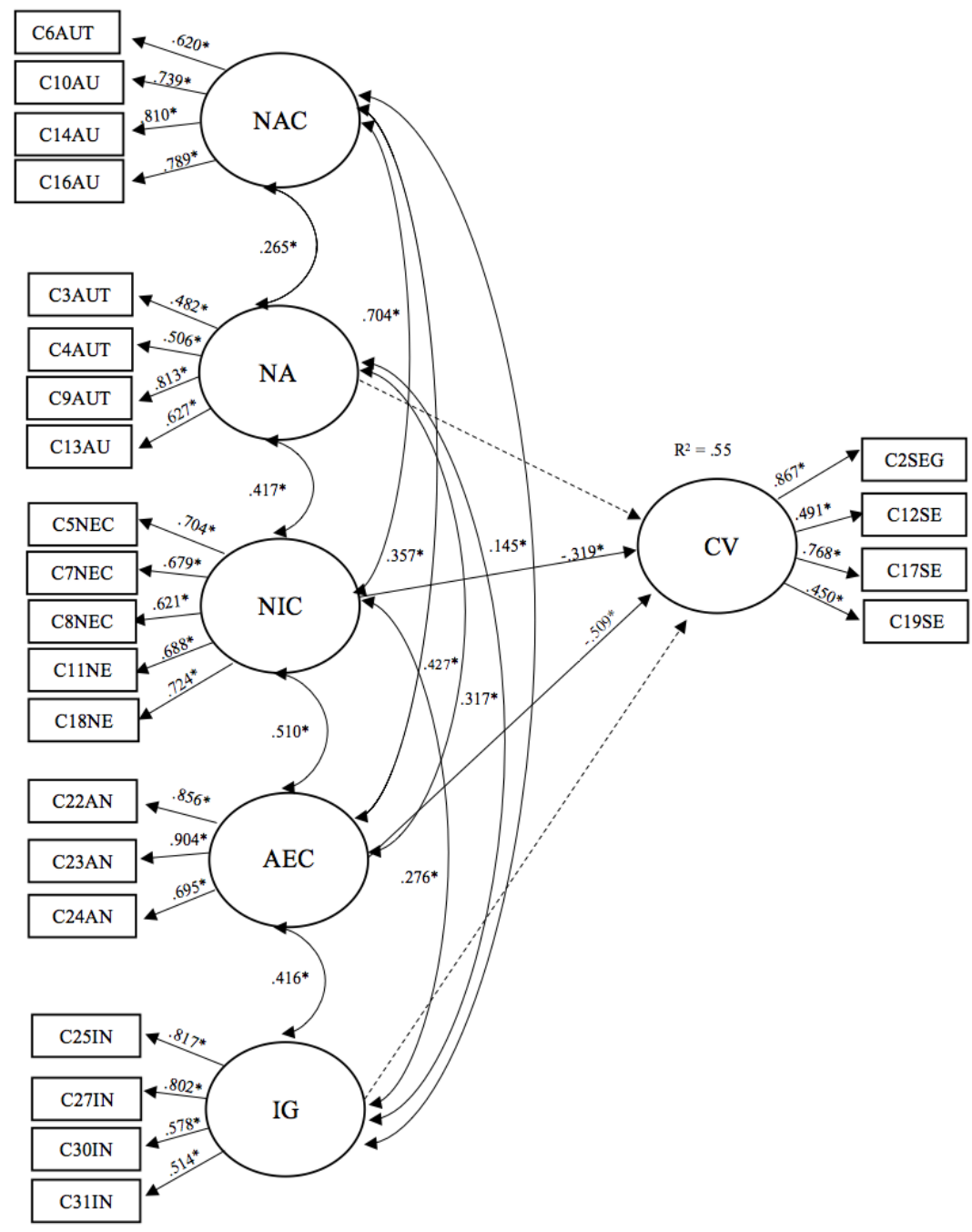

Clave: $\mathrm{CV}=$ certeza vocacional; $\mathrm{NAC}=$ necesidad de autoconocimiento; $\mathrm{NA}=$ Necesidad de autoeficacia; $\mathrm{NIC}=$ Necesidad de información de carrera; $\mathrm{AEC}=$ Ansiedad en elección de carrera; IG = Indecisión generalizada .

Figura 2. Modelo estructural de Certeza Vocacional en estudiantes universitarios del centro y sur de Sonora, México. $X^{2}=349(235 \mathrm{gl}) p=.000$. IBBANN $=.938$, IAC $=.947$, RMSEA $=.046(.035 ; .055)$. Las líneas punteadas indican relaciones no significativas 
También se encontraron correlaciones entre los diferentes factores de carrera, las cuales son consistentes con lo que teóricamente se esperaba. La certeza vocacional se explica en $55 \%$ de su variabilidad por este modelo de relaciones entre los factores de carrera, como fue medido en este estudio.

Los valores de bondad de ajuste fueron $\chi^{2}=349(235 g l) p=.000 ;$ IBBANN $=.93$, $\mathrm{IAC}=.94, \mathrm{RMSEA}=.04$ con un intervalo de confianza de .03 y .05 . Lo cual indica que el modelo sólo posee bondad de ajuste práctico. Durante el proceso de modelamiento estructural se eliminaron seis reactivos que no presentaron relaciones significativas y por no aportar a la bondad de ajuste del modelo (Bentler, 2006); salió un reactivo de la escala necesidad de autoconocimiento, dos reactivos de la escala certeza vocacional y tres reactivos de la variable latente indecisión generalizada, quedando el instrumento con un total 24 reactivos significativos.

\section{Discusión}

Se elaboró y probó un modelo estructural de certeza vocacional y factores de carrera que explica $55 \%$ de la variabilidad de la certeza vocacional, en estudiantes de dos ciudades del noroeste de México. El poseer instrumentos confiables y válidos para medir la certeza vocacional es una necesidad social señalada por investigadores como Gati y Levin (2014). Otro de los hallazgos importantes en este estudio fue el que las mujeres de la muestra adquirieron puntuacio- nes más altas en indecisión generalizada que los hombres, lo cual señala la necesidad de investigar el área de la decisión vocacional desde una perspectiva de género (De Garay, \& Del Valle, 2012). Otro dato a destacar es que los estudiantes que trabajan además de estudiar poseen menos indecisión generalizada que los que no trabajan, y que los estudiantes reubicados presentan mayor ansiedad ante la elección de carrera. Además, los estudiantes universitarios del centro del estado de Sonora reportan mayor necesidad de autoeficacia que los estudiantes del sur del estado. Asimismo, se observaron diferencias en los estudiantes de Psicología de ambas universidades, donde los estudiantes del centro del estado presentan medias más elevadas en necesidad de autoeficacia, necesidad de información de la carrera y ansiedad en la elección que los estudiantes de la universidad del sur del estado; respecto a certeza vocacional son los estudiantes de Psicología del sur de Sonora quienes presentan mayor certeza vocacional. No se encontraron diferencias entre los estudiantes de negocios y Administración de ambas instituciones. Finalmente, se observó una diferencia entre los estudiantes de Psicología y los del área de negocios de ambas universidades, respecto a la necesidad de información de la carrera, donde los estudiantes de negocios y Administración, reportaron mayor necesidad de información.

Estudios como el de Burns, Jasinski, Dunn y Fletcher (2013), Hsieh y Huang (2014) e Isik (2012) coinciden, a partir de sus respectivos resultados de investigación, en la importancia 
de la autoeficacia en la decisión de carrera. En este estudio la autoeficacia tiene un efecto indirecto hacia la certeza vocacional a través de la necesidad de información sobre la carrera y la necesidad de autoconocimiento, y dada la covarianza entre ellos, se puede afirmar que estos tres factores cognoscitivos están vinculados; se puede asumir que predicen la certeza vocacional. Este importante hallazgo convoca a tomar en cuenta el fomento del autoconocimiento y la autoeficacia en el proceso de decisión de carrera y no sólo brindar información acerca de las diferentes profesiones de la oferta educativa de la entidad federativa y/o el país.

Los hallazgos anteriores atañen a la recomendación de Gati y Levin (2014) acerca de emplear instrumentos que permitan facilitar la toma de decisión de carrera; aunado a ello, el modelo estructural resultante en este estudio muestra cuáles son los principales factores a tomar en cuenta para fomentar o apoyar en la decisión de carrera de los estudiantes que estén pasando por ese proceso. Contar con instrumentos que facilitan la toma de decisión vocacional permitirá optimizar los recursos destinados a la atención del estudiantado y facilitará un desempeño óptimo en la formación profesional.

De igual manera, es importante la participación de instituciones educativas que atiendan bajo esta perspectiva de género algunos retos (Macías, 2016), en los cuales se considera la importancia de las diferencias de participación por niveles de estudio superiores, donde se señala que la mujer tiene una menor participación en estudios de doctorado y en investigación, por ejemplo; otro de estos retos que identifica Macías es lograr una participación equilibrada de hombres y mujeres en todas las áreas de estudio $\mathrm{y}$, por último, crear una vinculación con el ámbito laboral en donde se ofrezcan las mismas oportunidades para los profesionistas tanto hombres como mujeres.

Es importante señalar algunas de las limitaciones de la presente investigación, donde se trabajó con una muestra no aleatoria y que utilizó como método de recolección de datos, el autorreporte; no obstante, se obtuvo la confiabilidad de los instrumentos, así como la validez de las escalas a través de un análisis factorial confirmatorio, por modelamiento de ecuaciones estructurales.

Por último, los hallazgos de este estudio pueden contribuir al rediseño de los materiales de estudio correspondientes a las asignaturas de orientación vocacional, como lo señalan Velarde, González y Camarena (2013), así como al mejoramiento de las guías de asesoría vocacional utilizadas en el subsistema de bachillerato (Velarde, \& González, 2013) y en la atención de los estudiantes universitarios, sobre todo a los de recién ingreso.

\section{Referencias}

Aguilar, J., Pacheco, J., Andrade, J., Vargas, J., Gutiérrez, M., \& Zetina, G. (1992). Estudio sobre la Validez Concurrente del Inventario de Factores de Carrera con Estudiantes de Licenciatura. México: UNAM. 
Aguilar, J., Peña, L., Pacheco, J., \& De la Paz, C. (1993). Adaptación y ampliación del inventario de factores de carrera. Investigación Psicológica, 3, 53-63.

Bentler, P. (2006). EQS, Structural Equations Program Manual. Los Angeles: BMDP Statistical Software.

Bulgarelli, R., Rivera, J., \& Fallas, M. (2016). El proceso vocacional del estudiantado universitario en condición de logro y rezago académico: Un análisis desde el enfoque evolutivo de Donald Super. Revista Electrónica Educare, 21(1), 1-24.

Burns, G. N., Jasinski, D., Dunn, S., \& Fletcher, D. (2013). Academic Support Services and Career Decision-Making Self-Efficacy in Student Athletes. The Career Development Quarterly, 61(2), 161-167.

Canto, J. (2000). Certeza de elección de carrera y preferencia vocacional. Revista Educación y Ciencia, 4(21), 43-55.

Chartrand, J., Robbins, S., Morrill, W., \& Boggs, K. (1990). Development and validation of the career factors inventory. Journal of Counseling Psychology, 37(4), 491-501.

De Garay,A., \& Del Valle, G. (2012). Una Mirada a la presencia de las mujeres en la educación superior en México. Revista Iberoamericana de Educación Superior, 6(3), 1-30.

Fernández, F. (2003). El estudio sociológico de la educación. En F. Fernández (Ed.), Sociología de la Educación (pp. 1-34). México: Pearson Prentice Hall.
Fuentes, M. (2010). La orientación profesional para elegir fundamentadamente una ocupación: Propuesta alternativa. Revista Mexicana de Psicología, 27(2), 237-246.

Fuentes-Vega, M. (2016). Efecto de la certeza vocacional y las relaciones familiares en la elección de carrera de estudiantes de bachillerato (Tesis de maestría inédita). Universidad de Sonora.

Gati, I., \& Levin, N. (2014). Counseling for Career Decision-Making Difficulties: Measures and Methods. The Career Development Quarterly, 62(2), 98-113.

González, D., \& Maytorena, Ma. A. (2005). Modelo estructural de factores de carrera, seguridad vocacional y esfuerzo académico. Revista Interamericana de Psicología, 39(1), 39-48. ISSN: 0034-9690. Brasil.

González, D., \& Maytorena, Ma. A. (2007). Decisión vocacional en estudiantes de bachillerato y de educación superior. Quehacer cientifico en Chiapas, 1(3), 40-45. Recuperado de http://www.dgip.unach.mx/images/ pdf-REVISTA-QUEHACERCIENTIFICO/ QUEHACER-CIENTIFICO-2007-ener-jun/ decision_vocacional_en_estudiantes_de_ bachillerato.pdf

Hou, C., Wu, L., \& Liu, Z. (2013). Parental emotional warmth and career decision-making difficulties: A model of intellectual-cultural orientation and conscientiousness. Social Behavior and Personality: an international journal, 41(8), 1387-1397. 
Hsieh, H. H., \& Huang, J. T. (2014). The Effects of Socioeconomic Status and Proactive Personality on Career Decision Self-Efficacy. The Career Development Quarterly, 62(1), 29-43.

Işik, E. (2012). The relationship of career decision self-efficacy, trait anxiety, and affectivity among undergraduate students 1 . Psychological Reports, 111(3), 805-813.

Kim, C., Kang, M., \& Kim, Y. (2013). Effect of Conflictual Independence from and Attachment to Mother on Career Indecision: The Mediating Effect of Indecisiveness and Relative Effect Sizes of the Two Independent Variables. Journal of Asia Pacific Counseling, 3(2), 151-164.

León, A. (2016). Deserción escolar en una universidad al sur de Sonora (Tesis de maestría). Instituto Tecnológico de Sonora, México. Recuperado de http://sib2.itson.mx/cgi-bin/ koha/opac-detail.pl?biblionumber=74418.

Macías, G. (2016). Mujeres y hombres en la universidad. Tendencias de participación en la matrícula y desafíos actuales de la educación superior. Revista educ@rnos, 5(2021) 93-114.

Mariñez, V., \& González, D. (2013). Material Didáctico Innovador: evaluación y diseño. Colección Universitaria. México: Orfila Valentini-Universidad de Sonora.

Velarde, D., \& González, D. (2013). Influencia de factores personales y sociales sobre la certeza vocacional. Psicumex, 3(2), 15-32.
Velarde, D., González, D., \& Camarena, B. (2013). Certeza vocacional en el contexto universitario. México: Editorial Orfila Valentini-Universidad de Sonora.
Revisado: 18/06/2018

Aceptado: 30/07/2018 